\title{
The processing of deviant information in prediction and evaluation
}

\author{
IRWIN P. LEVIN, JOHN R. IMS, JOHN C. SIMPSON, and KYUNG JA KIM \\ University of Iowa, lowa City, lowa 52242
}

\begin{abstract}
Analyses of information integration and of retention were used to examine the processing of deviant information in prediction and evaluation tasks. Sets of test scores were presented serially for a group of hypothetical students, and subjects were asked to evaluate the performance of each student or predict each student's performance on a comprehensive final exam. An averaging model with greater weight for the more recent scores than for the earlier scores was supported for both types of task, but the recency was more pronounced in the prediction task. Weighting of deviant scores differed in the prediction and evaluation tasks. Significant discounting (underweighting) of deviant scores was obtained only in the prediction task. The ability to recall deviant scores on uncued tests of retention was higher in the prediction task than in the evaluation task. Prediction of future performance based on inconsistent measures of past performance thus appears to be an active process involving the discovery and discounting of unrepresentative information.
\end{abstract}

A common theme in recent reviews of human judgment research is the move from normative theory, with its emphasis on comparisons of actual to optimal behavior, to descriptive models, with concomitant emphasis on psychological interpretations of observed behavior (Anderson, 1974; Kahneman \& Tversky, 1973; Slovic, Fischhoff, \& Lichtenstein, 1977; Slovic \& Lichtenstein, 1971). An interesting question in the formulation of models of human judgment is how deviant pieces of information are processed. The present study uses descriptive models to provide a framework for examining the processing of deviant information in situations involving prediction and evaluation.

Kahneman and Tversky (1973) studied "the psychology of prediction" and concluded that people violate the logic of statistical prediction by disregarding factors such as information reliability and prior outcome probability, while relying almost exclusively on the heuristic of "representativeness." According to these authors, people predict by selecting the outcome that is most representative of the input, as if the input information were error free. In their studies, predictions and evaluations tended to coincide. But what if the input information contains inconsistencies that call to question the reliability of the information? The present hypothesis is that the integration of information under such circumstances will differ for predictions and evaluations.

The current prediction task is one in which subjects are given a set of test scores for each of a series of hypothetical students and are asked to predict each

Requests for reprints should be addressed to Irwin P. Levin, Department of Psychology, University of Iowa, Iowa City, Iowa 52242 . student's performance on a comprehensive final examination. The evaluation task is one in which subjects are asked to rate the performance of each hypothetical student. In some cases, the set of scores for a given student includes one score that is deviant from the rest. A single deviant score is apt to be viewed as a valid measure of current performance but as an unrepresentative measure of a person's capabilities. Thus, the deviant score is expected to be weighted less in predicting future performance than in evaluating current performance.

Description of a recent study by Levin (1976b) will help to lay the groundwork for testing this hypothesis. The study used the "intuitive statistics" paradigm (Peterson \& Beach, 1967) for examining human inferences based on fallible information. The usefulness of this paradigm for studying information integration processes has been described elsewhere (Anderson, 1964, 1968; Birnbaum, 1976; Levin, 1974, 1975; Levin, Kim, \& Corry, 1976). In Levin's (1976b) study, subjects were asked to make judgments about each of a series of sets of numerical scores. Some of these sets contained an "outlier" score that was deviant from the rest. In the "descriptive condition," subjects were asked to estimate in a brief time interval the mean or average value of the scores presented in each set, and in the "inference condition," subjects were asked to infer the mean of the population from which the set of scores represented a random sample. The weighting of deviant scores was assessed by comparing responses to sets containing a high deviant score with responses to sets containing a low deviant score. The deviant scores were weighted at least as much as other scores in forming a descriptive judgment. But deviant scores were weighted less or discounted in forming an 
inference. Levin concluded that subjects will discount a deviant score in making an inference from a sample to a population when they recognize such a score to be unrepresentative of the population. It can be inferred from Levin's results that subjects are most apt to recognize a score as deviant when it is at least twice as far from the mean of the set as any other score and when all the scores in a set are presented simultaneously.

The present study compares the integration of information in prediction and evaluation tasks using an extension of the methodology of Levin's (1976b) study. If the distinction between evaluation and prediction is analogous to the distinction between descriptive and inference judgments, then similar differences in the processing of deviant information can be expected. Such an expectation seems reasonable because evaluations and descriptions should be confined to input information, whereas predictions and inferences involve extrapolations beyond the input data.

In addition, this study provides an initial attempt to develop a method for measuring how subjects attend to the various components of an information integration task. The method involves a combination of information integration methodology (Anderson, 1974) and measures of retention. A recall task was used in conjunction with an information integration task in a study by Anderson and Hubert (1963), but, in contrast to the present methods, their subjects were forewarned that they would have to recall the material presented. The purpose of that study was to see how the manipulation of attentional factors through recall tests would change order effects in information integration.

In order to use recall measures to reflect attentional processes in information integration without actually intruding on these processes, subjects in the present study were not forewarned that they would be tested for recall. Subjects were given a series of sets of eight numbers each, where the numbers in a set were said to be a succession of test scores for a student. Some subjects were told to rate (evaluate) the performance of each student in relation to the other students, and some subjects were asked to predict the performance of each student on a subsequent comprehensive final exam. Following a number of such rating trials or prediction trials, subjects were given additional trials where they were asked (without precuing) to recall the scores they had just seen or estimate the trend present in the scores. The retention measures should then provide evidence on how the subjects were using information in the judgmental (integration) tasks.

\section{METHOD}

\section{Subjects}

The subjects were 160 students from introductory psychology classes at the University of Iowa. Subjects were tested in groups of 7 to 10.

\section{Stimuli}

Subjects were given a series of sets of eight numbers each, where the numbers in a set were said to be a succession of test scores, expressed as percent correct answers, for a given student in a particular class. Each different set was said to represent the scores for a different student in the class. Some of the sets contained a single deviant score and some did not. Nine of the sets were formed by varying the mean of the first four scores and the mean of the second four scores. In these sets, all scores in each half of the set were within 10 points of the mean of that half. Within this restriction, scores were chosen from a table of random numbers, thus generating approximately rectangular distributions, and constants were added to achieve the desired mean values. The following is an example of a set where the mean of the first four scores (M1) is 60 , the mean of the second four scores (M2) is 70 , and there is no single deviant score: $62,52,66,60,78,63,72,67$. Each of four additional sets contained a single deviant score. The deviant score was between 24 and 26 points (or approximately 6 SD units when the standard deviation was computed from the remaining seven scores) above or below the mean of the set. The following is an example of a set with a high deviant score: $60,64,50,56,58$, $86,54,52$.

\section{Experimental Design}

The nine sets which did not contain a deviant score were constructed according to a 3 by 3 factorial design where each of three values for the mean of the first four scores $(M 1=50$, 60 , or 70) was combined with each of three values for the mean of the last four scores (M2 $=50,60$, or 70 ). The four sets that contained a deviant score were constructed according to a 2 by 2 factorial design where the direction of the deviant score (above or below the mean of the set) and the position of the deviant score (in the first half or the last half) were varied. Each of these four sets had a mean of 60 . While the data from the 3 by 3 factorial design for sets without deviant scores and the data from the 2 by 2 factorial design for sets with deviant scores were analyzed separately, these different sets were interspersed randomly over trials. Practice trials and end anchors (sets with extreme values of $M 1$ and $M 2$ ) were also included to bring the total number of trials on the information integration task to 27 .

Four groups of 40 subjects each were chosen according to a randomized blocks design. The four groups are labeled the "rating group," the "rating plus trend group," the "prediction group," and the "prediction plus discounting group," in accordance with the instructions they received for the information integration task (see below).

\section{Procedure}

Subjects were asked to rate or predict the performance of each hypothetical student using a $20-\mathrm{cm}$ graphic (line-mark) rating scale labeled "very good" and "very bad" at the extremes, but otherwise unmarked. This scale was reproduced on each page of a response booklet. Subjects responded on a given trial by placing a slash mark somewhere along the scale on the appropriate page. Responses were measured and converted to a 20-point scale with higher numbers representing more favorable responses.

Subjects in the rating group were told to rate the performance of each student and to consider each score to be of equal importance. Subjects in the rating plus trend group were specifically told to consider in their ratings whether or not the student showed improvement over the course of the semester. The subjects in the prediction group were asked to predict the performance of each student on a comprehensive final exam. The subjects in the prediction plus discounting group were also asked to predict the performance of each student on a final exam. In addition, the latter subjects were told that occasionally a student will have an unusually "good" or "bad" day and that such scores may not necessarily be good predictors of subsequent performance. This additional cue concerning the unrepresentativeness of deviant scores was designed to promote the discounting of such scores. 
The numbers in a set were displayed serially. Each number was printed on a separate slide, each slide was presented for $3 \mathrm{sec}$ on a Carousel projector, and a 7 -sec response interval was allowed. Numbers were ordered such that the number on the first slide was said to represent the first test of the semester, the second slide represented the second test, and so on.

Retention questions. Following the 27 initial information integration trials, three additional trials were included where retention as well as integration of information was required. On each of these trials, the subjects in a given group made the usual rating or prediction response appropriate to that group and were then asked a question concerning the scores presented on that trial. The subjects were given $30 \mathrm{sec}$ to respond to the question. On the first two of these last three trials, the subjects were not forewarned that their rating or prediction response would be followed by a retention question.

Half the subjects in each group were given a trend question first, and the other half were given a recall question first. The trend question was asked immediately after the subjects made their rating or prediction response to a set of scores in which $M 1=74$ and $M 2=62$. The subjects were asked to indicate whether the first half or the second half of the scores was higher. The recall question was asked immediately after the subjects made their rating or prediction response to a new set of scores containing a deviant score. The subjects were asked to write down as many of the numbers in the set as they could recall in any order.

A second recall question was included on the final trial of the experimental session where subjects were told ahead of time that they would be asked to recall the scores in the next set following their rating or prediction response. This question was designed to provide a basis for comparing recall with and without precuing and for determining if the four different groups were comparable in retention ability.

\section{RESULTS AND DISCUSSION}

Analysis of the data was divided into three parts: analysis of judgmental responses (ratings and predictions) to sets without deviant scores, analysis of judgmental responses to sets that contained a deviant score, and analysis of retention measures.

\section{Responses to Sets Without Deviant Scores}

Figure 1 shows the mean response for each combination of $\mathrm{M} 1$ and $\mathrm{M} 2$ for each group. A 3 by 3 repeated-measures analysis of variance was performed on these data for each group. These results are summarized in Table 1 in terms of the mean squares, $F$ ratios, and weight estimates.

The near parallelism of the lines in the upper lefthand panel of Figure 1 suggests that an additive model can adequately describe the data for the rating group. Corresponding to this, the analysis of variance showed that, while $M 1$ and $M 2$ were significant sources of variance, the interaction did not approach statistical significance. It can be seen in Table 1 that the weight for $M 2$ was greater than the weight for $\mathrm{M} 1$, suggesting a recency effect. The reliability of the recency effect was examined by comparing the effects of variations in $\mathrm{M} 1$ and $\mathrm{M} 2$. For each subject the range of marginal means for variations in M1 was subtracted from the range of marginal means for variations in M2. For subjects in the rating group, the resulting mean
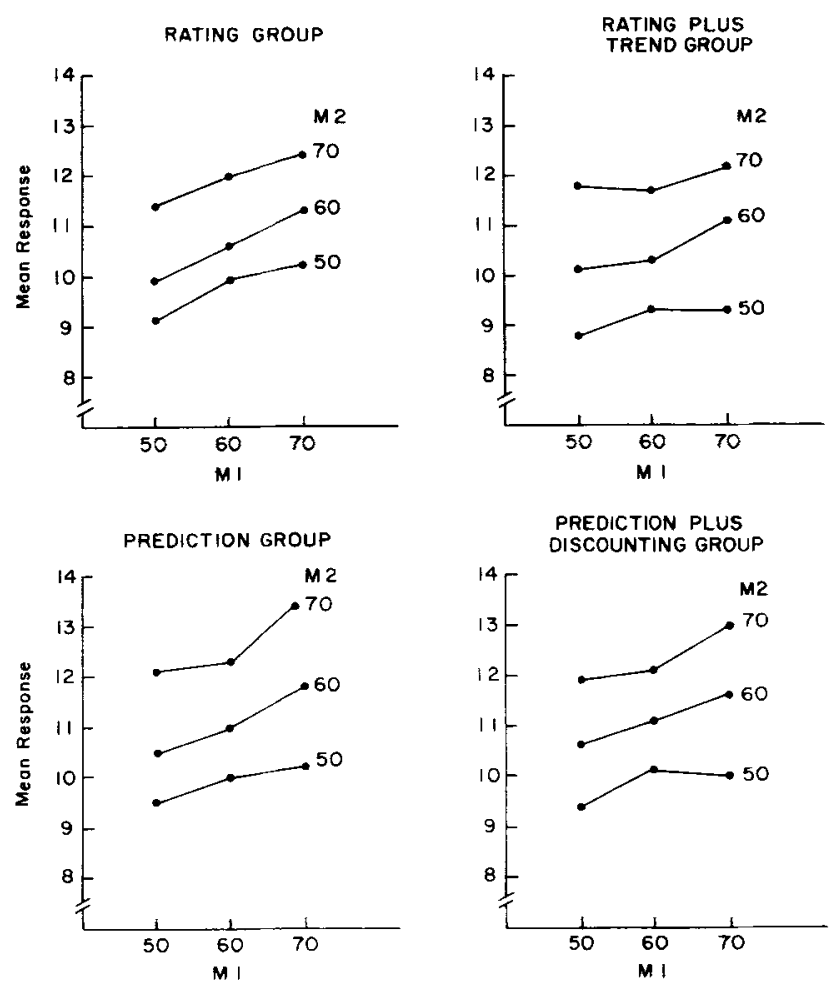

Figure 1. Mean responses to sets without deviant scores.

difference score was significantly greater than zero $[F(1,39)=17.82, \quad p<.01]$, showing a significant recency effect.

Results for the rating group are consistent with the predictions of a constant weight averaging model where weights are constant within levels of $\mathrm{M} 1$ and $\mathrm{M} 2$ but where $\mathrm{M} 2$ has greater weight than $\mathrm{M} 1$. In a similar task with simultaneous presentation of scores (Levin, Note 1), the weights for $\mathrm{M} 1$ and $\mathrm{M} 2$ were equal. Nevertheless, as can be seen by comparing the weight estimates in Table 1, the recency found for the rating group was less than the recency for any other group. This can also be seen in Figure 1 by noting that the separation of lines for different levels of M2 is least for the rating group.

Results for the rating plus trend, prediction, and prediction plus discounting groups were similar to each other. Both $M 1$ and $M 2$ were significant sources of variance in each case. The M1 by M2 interaction was not statistically significant for the rating plus trend group or the prediction group. However, there was a small but statistically significant interaction for the prediction plus discounting group, which can be attributed to a disproportionately low mean response to $\mathbf{M} 1=70, \mathbf{M} 2=50$. Perhaps the subjects in this group tended to discount the higher scores in this set. Despite this single small interaction effect, the constant weight averaging model appears to provide a good description of the process by which the scores were integrated. (See 
Table 1

Analy sis of Variance Results for Sets Without Deviant Scores

\begin{tabular}{|c|c|c|c|}
\hline & $\begin{array}{c}\text { Mean } \\
\text { Square }\end{array}$ & F Ratio & $\begin{array}{c}\text { Estimated } \\
\text { Weight }\end{array}$ \\
\hline $\begin{array}{l}\text { Rating Grou } \\
\text { M1 } \\
\text { M2 } \\
\text { M1 by M2 }\end{array}$ & $\begin{array}{r}40.72 \\
140.55 \\
.75\end{array}$ & $\begin{array}{l}F(2,78)=37.54^{*} \\
F(2,78)=144.98^{*} \\
F(4,156)=1.08\end{array}$ & $\begin{array}{l}.35 \\
.65\end{array}$ \\
\hline $\begin{array}{l}\text { Rating Plus } \\
\text { M1 } \\
\text { M2 } \\
\text { M1 by M2 }\end{array}$ & $\begin{array}{r}\text { nd Group } \\
11.44 \\
227.57 \\
2.09\end{array}$ & $\begin{array}{l}F(2,78)=7.37^{*} \\
F(2,78)=150.03^{*} \\
F(4,156)=1.85\end{array}$ & $\begin{array}{l}.17 \\
.83\end{array}$ \\
\hline $\begin{array}{l}\text { Prediction } G \\
\text { M1 } \\
\text { M2 } \\
\text { M1 by M2 }\end{array}$ & $\begin{array}{r}42.04 \\
216.08 \\
1.57\end{array}$ & $\begin{array}{l}F(2,78)=44.50^{*} \\
F(2,78)=138.19^{*} \\
F(4,156)=1.91\end{array}$ & $\begin{array}{l}.29 \\
.71\end{array}$ \\
\hline $\begin{array}{l}\text { Prediction } P 1 \\
\text { M1 } \\
\text { M2 } \\
\text { M1 by M2 }\end{array}$ & $\begin{array}{c}\text { Discount } \\
24.22 \\
190.65 \\
2.65\end{array}$ & $\begin{array}{l}\text { Group } \\
F(2,78)=24.39^{*} \\
F(2,78)=182.99^{*} \\
F(4,156)=3.57^{*}\end{array}$ & $\begin{array}{l}.26 \\
.74\end{array}$ \\
\hline
\end{tabular}

Note-See Levin et al. (1976) for a description of the method of estimating,weights.

${ }^{*} p<.01$

Levin et al., 1976, for a critical test supporting the averaging model with similar stimuli.) The dominant result for the rating plus trend, prediction, and prediction plus discounting groups was a strong recency effect $[F(1,39)=29.13,34.89$, and 32.40 , respectively, for these groups]. Consistent with the results of the analogous study using simultaneous presentation of scores (Levin, Note 1), recency effects were greater for predictions than for evaluations, unless subjects were specifically instructed to consider trends in their evaluations.

\section{Responses to Sets With Deviant Scores}

Responses to the sets containing a deviant score (all of which had a mean of 60 ) were analyzed with a 2 by 2 repeated-measures analysis of variance in which the factors were position of deviant score (first half vs. second half) and direction of discrepancy (positive when the deviant score was the highest score in the set and negative when the deviant score was the lowest score in the set). Discounting of deviant scores is evidenced when responses to sets with positive deviant scores are lower than responses to sets with negative deviant scores. This is because the remaining scores are relatively low in a set with a high deviant score and the remaining scores are relatively high in a set with a low deviant score. Reliable discounting effects can thus be inferred when the mean response to sets with positive deviant scores is significantly lower than the mean response to sets with negative deviant scores.

Mean responses to sets with deviant scores are plotted in Figure 2. It can be seen that responses to sets with positive (+) deviant scores tend to be higher than responses to sets with negative $(-)$ deviant scores in the rating group (upper left-hand panel of Figure 2). This is the opposite of discounting and represents overweighting of deviant scores, but the overweighting tendency did not reach statistical significance. These results are consistent with those of earlier studies of personality impression formation in which evaluations based on inconsistent information could be described as an averaging of information values with little or no reduction in the weight of the inconsistent stimulus (Anderson \& Jacobson, 1965; Hendrick \& Costantini, 1970).

Tendencies to discount deviant scores can be seen in all other groups where responses to sets with positive deviant scores were lower than responses to sets with negative deviant scores. The discounting effect was not statistically significant for the rating plus trend group. The discounting effect was strongest in the prediction plus discounting group $[F(1,39)=23.61, p<.01]$, thus demonstrating that the subjects were able to detect deviant scores and comply with instructions that cued them that deviant scores are poor predictors of future performance. However, discounting effects in the prediction group are especially interesting since they occurred in the absence of discounting cues. In the prediction group, responses were lower to sets with positive deviant scores than to sets with negative deviant scores, but this difference reached statistical significance only when the deviant score occurred in the second half $[\mathrm{t}(39)=4.46, \mathrm{p}<.01]$. When viewed
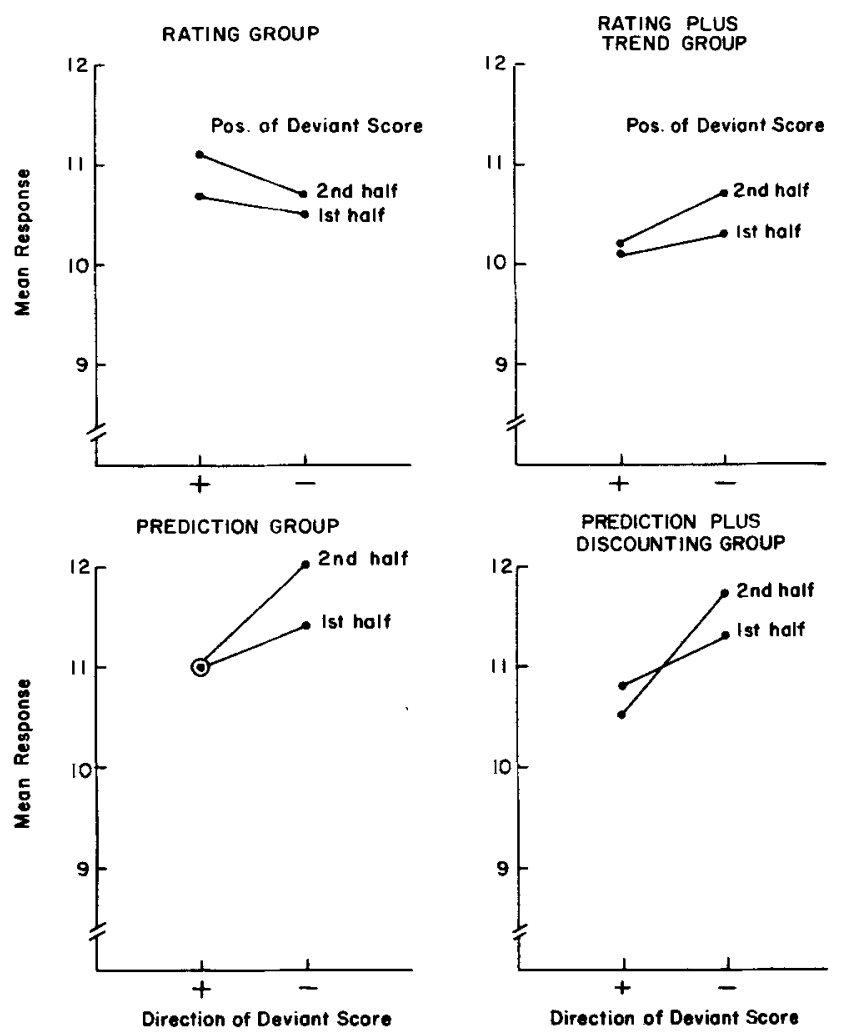

Figure 2. Mean responses to sets containing deviant scores. 
in light of the finding that scores in the second half are generally weighted more than scores in the first half when predicting future performance, this is consistent with the notion that discounting is an "active" process. This will be discussed further in a later section.

In summary, the rating group showed a small recency effect and a slight (but nonsignificant) tendency to overweight deviant scores. The rating plus trend group showed a large recency effect and a slight (but nonsignificant) tendency to discount deviant scores. The prediction and prediction plus discounting groups showed large recency effects and statistically reliable discounting of deviant scores.

\section{Retention Measures}

Responses to the three retention questions-trend recognition, recall without precuing, and recall with precuing-are summarized in Table 2 . It can be seen that the groups performed at a relatively high level on the trend question (recognizing that the first half of the scores on that trial were, on the average, higher than the second half of the scores). The prediction group did poorer than the other groups on this question, but a chi-square test comparing the proportion of correct responses on the trend question across groups did not reach statistical significance. While only the rating plus trend group was specifically instructed to examine trends in processing the scores, the studentsubjects might well have had a set to notice trends in exam scores even when that information was irrelevant to their judgments.

Mean number of correct responses on the recallwithout-precuing question did not differ significantly between the groups, nor did mean number of correct responses on the recall-with-precuing question. This shows that the groups were comparable on retention ability. Performance was approximately $50 \%$ better when subjects were cued that they would be asked to recall the scores in the forthcoming set than when they were not cued.

Of primary interest was retention of the deviant score in recall without precuing. Recall that there was a tendency to overweight deviant scores in the rating group and a tendency to discount deviant scores in the other groups. The rating group had a significantly poorer percentage of correct recall of deviant scores than did the other groups combined $\left[\chi^{2}(1)=4.97\right.$, $p<.05]$. Thus, there was a positive relationship between the tendency to discount deviant scores and the tendency to recall deviant scores. In all groups except the rating group, the probability of recalling the deviant score without precuing was greater than the probability of recalling a nondeviant score.

\section{SUMMARY AND CONCLUSIONS}

Making ratings and predictions based on sets without deviant scores could be described as a process of averaging information values. This is analogous to studies of number averaging which show that intuitive estimates of mean values can indeed be described by an averaging model (Anderson, 1964, 1968; Levin, 1975). The present results, along with those of Levin (1974, 1975) and Levin et al. (1976), extend the study of intuitive statistical processes by demonstrating that analogous intuitive statistical operations underlie a variety of affective judgments and decisions. This reinforces the descriptive (nonnormative) framework described earlier by showing that useful analyses of human judgmental processes need not rely on physical yardsticks of accuracy or optimality. (See also Levin, 1976a).

The recency found in the present study is not common to all studies of serial information integration (e.g., Levin, 1976b) and probably reflects the importance of trends in the stimuli of the present study. However, the recency effects found in the groups making predictions were considerably greater than those found in the rating group. Subjects respond as if the most recent performance measures are the best predictors of future performance. Not only is this a reasonable strategy, but it is consistent with the results of a variety of sequential inference and prediction tasks, ranging from "book bags and poker chips" tasks (Shanteau, 1970, 1972) to probability learning

Table 2

Summary of Responses on Retention Task

\begin{tabular}{|c|c|c|c|c|}
\hline & \multicolumn{4}{|c|}{ Group } \\
\hline & Rating & $\begin{array}{l}\text { Rating Plus } \\
\text { Trend }\end{array}$ & Prediction & $\begin{array}{l}\text { Prediction Plus } \\
\text { Discounting }\end{array}$ \\
\hline $\begin{array}{l}\text { Trend Question } \\
\text { Percent Correct }\end{array}$ & 80.0 & 82.5 & 55.0 & 72.5 \\
\hline $\begin{array}{l}\text { Recall Without Precuing } \\
\text { Mean Correct (Out of Eight) } \\
\text { Percent Correct on Deviant Scores }\end{array}$ & $\begin{array}{l}3.30 \\
27.5\end{array}$ & $\begin{array}{l}3.50 \\
47.5\end{array}$ & $\begin{array}{l}3.32 \\
47.5\end{array}$ & $\begin{array}{l}3.40 \\
47.5\end{array}$ \\
\hline $\begin{array}{l}\text { Recall With Precuing } \\
\text { Mean Correct (Out of Eight) } \\
\text { Percent Correct on Deviant Scores }\end{array}$ & $\begin{array}{l}5.22 \\
57.5\end{array}$ & $\begin{array}{l}5.35 \\
67.5\end{array}$ & $\begin{array}{l}5.25 \\
62.5 \\
\end{array}$ & $\begin{array}{l}5.65 \\
65.0\end{array}$ \\
\hline
\end{tabular}


(Friedman, Carterette, \& Anderson, 1968; Levin, Dulberg, Dooley, \& Hinrichs, 1972). In her review of such tasks, Jones (1971) supports an active information processing rather than a conditioning interpretation of the role of prior events in a prediction task. The present study also supports such an interpretation, with the evidence on the processing of deviant information being especially crucial in this regard.

Support for a constant weight averaging model in the processing of information in sets without deviant scores indicates that score weights depend on the serial position but not the absolute value of the individual score. Such, however, was not the case for deviant scores. Weights for deviant scores varied across groups. Weighting of the deviant score in sets with a single deviant score ranged from slight overweighting of deviant scores in the evaluations of the rating group to significant discounting (underweighting) of deviant scores in the prediction responses of the prediction and prediction plus discounting groups. The use of numerical stimuli rules out alternative interpretations, such as change of meaning, of the effects found here and supports the conceptualization of discounting as reduced weighting of deviant scores in the averaging process.

Discounting in the prediction group, without specific instructions to look for unrepresentative scores, is of particular interest because it distinguishes between the integration of information in forming evaluations and in making predictions. As expected, a deviant piece of information was weighted less in predictions than in evaluations. Since reliable discounting occurred only in the prediction task, discounting appears to be due to the perceived unrepresentativeness of a deviant item when predicting future events. A deviant item is, by definition, different from the rest; but whether or not it is treated differently depends on the use to be made of the information.

Retention of deviant scores was directly related to the tendency to discount deviant scores in the judgment task. This is analogous to the von Restorff effect in which an item that stands out in a list is recalled better than other items (Wallace, 1965). In the typical experimental test of the von Restorff effect, one item is isolated by presenting it, for example, in a different color than the others. By contrast, subjects in the present study had to discover that one score was inconsistent with the rest. Thus, "discounting" can be described as an active process of attending to and systematically deemphasizing (assigning reduced weight to) the deviant score. These findings rule out a simple monotonic relationship between attention level and information weighting, but they show that attentional processes underlie the differential weighting of different components of a set of information.

\section{REFERENCE NOTE}

1. Levin, I. P. Information integration, retention, and levels of information processing. Paper presented at the meeting of the American Psychological Association. Washington, D.C.. August 1976.

\section{REFERENCES}

Anderson, N. H. Test of a model for number-averaging behavior. Psychonomic Science, 1964, 1, 191-192.

Anderson, N. H. Averaging of space and number stimuli with simultaneous presentation. Journal of Experimental Psychology, 1968, 77, 383-392.

ANDERSON, N. H. Information integration theory: A brief survey. In D. H. Krantz, R. C. Atkinson, R. D. Luce, \& P. Suppes (Eds.), Contemporary developments in mathematical psychology (Vol. 2). San Francisco: Freeman, 1974.

Anderson, N. H., \& Hubert, S. Effects of concomitant verbal recall on order effects in personality impression formation. Journal of Verbal Learning and Verbal Behavior, 1963, 2. 379-391.

Anderson, N. H., \& Jacobson, A. Effects of stimulus inconsistency and discounting instructions in personality impression formation. Joumal of Personality and Social Psychology, 1965, 2, 531-539.

Birnbaum, M. H. Intuitive numerical prediction. American Journal of Psychology, 1976, 89, 417-429.

Friedman, M. P. Carterette, E. C. \& A Anderson, N. H. Longterm probability learning with a random schedule of reinforcement. Journal of Experimental Psychology, 1968, 78, 442-455.

Hendrick. C., \& Costantini, A. F. Effects of varying trait inconsistency and response requirements on the primacy effect in impression formation. Journal of Personality and Social Psychology, 1970, 15, 158-164.

Jones, M. R. From probability learning to sequential processing: A critical review. Psychological Bulletin, 1971, 76, 153-185.

Kahineman, D.. \& TVersky, A. On the psychology of prediction. Psychological Review, 1973, 80, 237-251.

LeVIN, I. P. Averaging processes in ratings and choices based on numerical information. Memory \& Cognition, 1974, 2. 786-790.

Levis, I. P. Information integration in numerical judgments and decision processes. Journal of Experimental Psychology: General. 1975, 104, 39-53.

Levin, I. P. Comparing different models and response transformations in an information integration task. Bulletin of the Psychonomic Society, 1976, 7, 78-80. (a)

LeVIN, I. P. Processing of deviant information in inference and descriptive tasks with simultaneous and serial presentation. Organizational Behavior and Human Performance, 1976, 15, 195-211. (b)

Levin, I. P., Dulberg, C. S., Dooley, J. F., \& Hinrichs, J. V. Sequential dependencies in single-item and multipleitem probability learning. Journal of Experimental Psychology, 1972, 93, 262-267.

LEVIN, I. P., KIM, K. J., \& CoRRY, F. A. Invariance of the weight parameter in information integration. Memory \& Cognition. 1976, 4, 43-47.

Peterson, C. R., \& Beach, L. R. Man as an intuitive statistician. Psychological Bulletin, 1967, 68, 29-46.

Shanteau, J. C. An additive model for sequential decision making. Journal of Experimental Psychology, 1970, 85, 181-191.

Shanteau, J. Descriptive versus normative models of sequential inference judgment. Joumal of Experimental Psychology. 1972, 93, 63-68.

Slovic, P.. Fischioff. B., \& Lichtenstein, S. Behavioral decision theory. Annual Review of Psychology, 1977, 28, 1-39.

Slovic, P.. \& Lichtenstein, S. Comparison of Bayesian and regression approaches to the study of information processing in judgment. Organizational Behavior and Human Performance, $1971,6,649-744$.

Wallace, W. P. Review of the historical, empirical, and theoretical status of the von Restorff phenomenon. Psychological Bulletin, 1965, 63, 410-424.

(Received for publication June 7, 1977; accepted September 16,1977,) 